\title{
Reliable Finite Element Methods for Self-adjoint Singular Perturbation Problems
}

\author{
Jean M.-S. LUBUMA* \\ Department of Mathematics and Applied Mathematics \\ University of Pretoria, Pretoria 0002, South Africa \\ E-mail: jean.lubuma@up.ac.za \\ Kailash C. PATIDAR \\ Department of Mathematics and Applied Mathematics \\ University of the Western Cape, Private Bag X17, Bellville 7535, South Africa \\ E-mail: kpatidar@uwc.ac.za
}

\begin{abstract}
It is well known that the standard finite element method based on the space $V_{h}$ of continuous piecewise linear functions is not reliable in solving singular perturbation problems. It is also known that the solution of a two-point boundary-value singular perturbation problem admits a decomposition into a regular part and a finite linear combination of explicit singular functions. Taking into account this decomposition, first, we design a finite element method (which we call Singular Function Method) where the space of trial and test functions is the direct sum of $V_{h}$ and the space spanned by these singular functions. The second method, based on the finite element discretization on a suitably refined mesh, is referred to as Mesh Refinement Method. Both of these methods are proved to be $\varepsilon$-uniformly convergent. Numerical examples which confirm the theory are presented.
\end{abstract}

AMS Subject Classification (2000): 65L10, 65L60, 65L70, 65L99.

Keywords : Singular perturbations, singular functions, finite element methods, boundary value problems, ordinary differential equations.

\section{Introduction}

We consider the self-adjoint singularly perturbed two-point boundary value problem

$$
\begin{gathered}
-\varepsilon\left(p(x) y^{\prime}\right)^{\prime}+q(x) y=f(x) \quad \text { on } \quad(0,1) \\
y(0)=0, \quad y(1)=0
\end{gathered}
$$

where $\varepsilon$ is a small positive parameter. Further, $f(x), p(x)$ and $q(x)$ are sufficiently smooth functions satisfying the boundedness and positivity conditions

$$
\widetilde{p} \geq p(x) \geq p^{*}>0 \quad \text { and } \quad \widetilde{q} \geq q(x) \geq q^{*}>0 .
$$

\footnotetext{
${ }^{*}$ Corresponding author
} 
Due to (1.2) and to the Lax-Milgram lemma, the problem (1.1) has a unique variational solution. That is, there exists a unique $y_{\varepsilon} \equiv y \in H_{0}^{1} \equiv H_{0}^{1}(0,1)$ such that

$$
a(y, v)=(f, v) \quad \forall v \in H_{0}^{1}(0,1)
$$

where the bilinear and the linear forms are given by

$$
a(y, v)=\int_{0}^{1}\left[\varepsilon p(x) y^{\prime}(x) v^{\prime}(x)+q(x) y(x) v(x)\right] d x
$$

and

$$
(f, v)=\int_{0}^{1} f(x) v(x) d x .
$$

Here, $H^{m}(0,1)$ denotes the Sobolev space ([1]) of order $m$ equipped with its usual norm whereas $H_{0}^{m}(0,1)$ is the closure in $H^{m}(0,1)$ of the space $\mathcal{D}(0,1)$ of infinitely differentiable functions on $(0,1)$ with compact support contained in $(0,1)$.

Problems in which a small parameter is multiplied to the highest derivative arise in various fields of science and engineering, such as fluid mechanics, fluid dynamics, elasticity, quantum mechanics, chemical reactor theory, hydrodynamics, etc. It is this feature of the differential equations that explains theoretically the physical phenomenon of boundary layers. Indeed, the main concern with singularly perturbed problems is the rapid and large variation of their solutions in one or more narrow "layer region(s)" where the solutions jump from one stable state to another or to prescribed boundary values. Such situations occur in many applications as highlighted in [10]. For instance, in physics, this happens in viscous gas flows in the zones near the boundary layers where the viscous flow jumps from the boundary values prescribed by the condition of adhesion to the inviscid flow or in the zones near the shock wave where the flow jumps from a subsonic to a supersonic state. Likewise, in chemical reactions, the rapid transition from one state to another is typical for solution processes, whereas population genetics in biology undergoe sudden changes. Apart from the concrete examples (e.g. diffusion-type problems, momentum conservations laws, etc), which are depicted in [10], we refer to [16] for further account on singular perturbation problems that arise in science and engineering.

The specific singular perturbation problem (SPP) (1.1) considered in this paper is dissipative in the sense that the rapidly varying component of the solution decays exponentially away from a localized breakdown or discontinuity point in the layer region(s) as $\varepsilon \rightarrow 0$. Consequently, classical methods always fail in providing reliable numerical results (in the sense that the parameter $\varepsilon$ and the mesh size $h$ cannot vary independently).

There are essentially two strategies to design schemes which have small truncation errors inside the layer region(s). The first approach, which forms the class of fitted mesh methods, consists in choosing a fine mesh in the layer region(s). The second approach is in the context of the fitted operator methods in which the mesh remains uniform but the difference schemes reflect the qualitative behavior of the solution(s) inside the layer region(s). A discussion using one or both of the above strategies can be found in in Miller et al. [17]. Some other relevant works may be found in the survey paper [8].

While finite difference methods that fall under both of the above categories have been discussed by the authors in $[12,13,14,15,18,19]$, this paper deals with the finite element method (FEM). However, the FEMs proposed here are specific in several respects, compared to the approach in for example $[6,9,11,21]$. Indeed based on the decomposition of the solution into a regular and a singular components given in [4], we construct more appropriate singular functions. Then we consider the direct sum of the space spanned by these 
singular functions with the usual space of piecewise continuous linear basis functions. The finite element method thus obtained is termed as Singular Function Method (SFM). It falls under the category of fitted operator methods. On the other hand, we use the Galerkin discretization on a non-uniform mesh which is suitably refined. This second finite element method is termed as Mesh Refine Method (MRM). Both of these methods are proved to be $\varepsilon$-uniformly convergent.

The advantage of the finite element method is the better possibilities of its extensions to higher dimensional problems. In this regard, the papers [7, 23] can be mentioned. Notice also that the initial setting that Babuska [2] and Strang and Fix [22] considered for the MRM and the SFM was that of domain with corners. The extension of the MRM and SFM to the singular perturbation problems considered in this paper has, to the authors' best knowledge, not been done in the literature. It is worthwhile undertaking this study because the nature of singularities of SPPs is completely different from those of problems with corners. However, we have restricted the study to the one-dimensional situation in order to simplify the exposition of the methods and to have explicit results. Another advantage of the finite element method over the finite difference method is that the latter is a particular case of the former whenever suitable quadrature formulae are used for the computation of the entries of the stiffness matrix and of the load vector (see $[3,20])$.

The rest of the paper is organized as follows. In Section 2, we describe the qualitative propeties of the solution of (1.1) in terms of the singular functions which are the founding stones for the SFM derived in Section 4. Section 3 deals with the standard finite element discretization. Using suitable interpolation error estimates, the MRM is derived in Section 5. The numerical implementation of the methods is described in Section 6. Finally in Section 7 , we draw some conclusions and indicate our future plans.

\section{Qualitative Behavior of the Solution}

The numerical methods in this paper are based on the following qualitative behavior of the solution $y(x)$ :

Theorem 2.1 Assume that $f(x) \in C^{0}[0,1]$ and $q(x) \in C^{2}[0,1]$ so that $y(x)$ is of class $C^{2}[0,1]$. Then the solution $y(x)$ admits the decomposition

$$
y(x)=\gamma_{0} S_{0}(x)+\gamma_{1} S_{1}(x)+g(x)
$$

where:

- $\gamma_{0}$ and $\gamma_{1}$ are real numbers

- $g(x)$ is the regular part which satisfies

$$
\left|g^{(k)}(x)\right| \leq C\left(1+\varepsilon^{1-\frac{k}{2}}\right), \quad \text { for } \quad k=0,1,2,
$$

and $C$ denotes here and after various constants, which are independent of $\varepsilon$ and the mesh size $h$.

- The functions

$$
S_{0}(x)=\exp \left(-x \sqrt{\frac{q(0)}{\varepsilon}}\right)-1+x-x \exp \left(-\sqrt{\frac{q(0)}{\varepsilon}}\right)
$$


and

$$
S_{1}(x)=\exp \left(-(1-x) \sqrt{\frac{q(1)}{\varepsilon}}\right)-x-(1-x) \exp \left(-\sqrt{\frac{q(1)}{\varepsilon}}\right)
$$

are the singular functions in the sense that

$$
\sup _{0<\varepsilon \leq 1} \sup _{x \in[0,1]}\left|S_{i}^{(k)}(x)\right|=\infty \text { for } k=1,2 \text { and } i=0,1 \text {. }
$$

Proof. It is shown in [4] that the solution of (1.1) admits the representation

$$
y(x)=\gamma_{0} \widetilde{S}_{0}(x)+\gamma_{1} \widetilde{S}_{1}(x)+\widetilde{g}(x)
$$

with constants $\gamma_{0}$ and $\gamma_{1}$, the singular functions

$$
\begin{gathered}
\widetilde{S}_{0}(x)=\exp \left(-x \sqrt{\frac{q(0)}{\varepsilon}}\right), \\
\widetilde{S}_{1}(x)=\exp \left(-(1-x) \sqrt{\frac{q(1)}{\varepsilon}}\right)
\end{gathered}
$$

and the regular part $\widetilde{g}(x)$ satisfying

$$
\left|\widetilde{g}^{(k)}(x)\right| \leq C\left(1+\varepsilon^{1-\frac{k}{2}}\right), \text { for } k=0,1,2 .
$$

Since we are dealing with a homogeneous boundary value problem, we modify the decomposition (2.1) into another one, with singular functions that take the values 0 at the end points of the interval. To this end, we consider the linear Lagrange interpolations

$$
P_{0}(x)=1-x+x \exp \left(-\sqrt{\frac{q(0)}{\varepsilon}}\right)
$$

and

$$
P_{1}(x)=x+(1-x) \exp \left(-\sqrt{\frac{q(1)}{\varepsilon}}\right)
$$

of the functions $\widetilde{S}_{0}(x)$ and $\widetilde{S}_{1}(x)$ at the nodes 0 and 1 . Now, adding and subtracting the functions $P_{0}(x)$ and $P_{1}(x)$ in $(2.1)$, we obtain the desired decomposition.

Remark 2.2 Theorem 2.1 shows that the solution $y(x)$ is singular in the sense that

$$
\sup _{0<\varepsilon \leq 1} \sup _{x \in[0,1]}\left|y^{(k)}(x)\right|=\infty \quad \text { for } \quad k=1,2 .
$$

Moreover, it is proved in [17] that

$$
\left|y^{(k)}(x)\right| \leq C\left(1+\frac{\exp \left(-x \sqrt{q^{*} / \varepsilon}\right)+\exp \left(-(1-x) \sqrt{q^{*} / \varepsilon}\right)}{\varepsilon^{k / 2}}\right), k=0,1,2 .
$$




\section{The Standard Finite Element Method}

Consider a uniform partition:

$$
0=x_{0}<x_{1}<\ldots<x_{n-1}<x_{n}=1
$$

and denote by $h$ the mesh width of a single element, i.e.,

$$
h=x_{j+1}-x_{j}, \forall j=0(1) n-1 .
$$

Let $V_{h} \subset H_{0}^{1}$ be the classical finite element space defined by

$$
V_{h}=\left\{y_{h} \in C^{0}[0,1],\left.y_{h}\right|_{\left[x_{i}, x_{i+1}\right]} \text { is linear , } y_{h}(0)=y_{h}(1)=0\right\} \text {. }
$$

We refer to $[3,20]$ for details on finite elements. Then the standard finite element method (FEM), i.e., the Galerkin discrete formulation corresponding to (1.3) reads: seek $y_{h} \in V_{h}$ such that

$$
a\left(y_{h}, v_{h}\right)=\left(f, v_{h}\right) \quad \forall v_{h} \in V_{h} .
$$

From Céa's lemma, we have

$$
\left\|y-y_{h}\right\|_{H^{1}} \leq \sqrt{\frac{M}{\alpha}} \inf _{v_{h} \in V_{h}}\left\|y-v_{h}\right\|_{H^{1}}
$$

where

$$
M=\widetilde{p} \max \{\varepsilon, \widetilde{q} / \widetilde{p}\}
$$

is an upper bound of the norm of $a(.,$.$) and$

$$
\alpha=p^{*} \min \left\{\varepsilon, q^{*} / p^{*}\right\}
$$

is the coercivity constant for $a(.,$.$) . As \varepsilon$ is very small, we note that the constant $M$ does not depend on $\varepsilon$ whereas $\alpha=p^{*} \varepsilon$. This fact will be implicitly used in what follows.

Denoting by $\Pi_{h}$ the global $V_{h}$-interpolation operator, it follows from (3.3) and by the interpolation theory in Sobolev spaces that

$$
\left\|y-y_{h}\right\|_{H^{1}} \leq \frac{C}{\sqrt{\varepsilon}}\left\|y-\Pi_{h} y\right\|_{H^{1}} \leq \frac{C h}{\sqrt{\varepsilon}}\left\|y^{\prime \prime}\right\|_{L^{2}} .
$$

Now since $C^{0}[0,1]$ is continuously embedded in $L^{2}(0,1)$, ( 3.4) along with (2.2) yield

$$
\left\|y-y_{h}\right\|_{H^{1}} \leq \frac{C h}{\sqrt{\varepsilon}} \sup _{x \in[0,1]}\left(1+\frac{\exp \left(-x \sqrt{q^{*} / \varepsilon}\right)+\exp \left(-(1-x) \sqrt{q^{*} / \varepsilon}\right)}{\varepsilon}\right) .
$$

For convenience, we write $(3.5)$ as

$$
\left\|y-y_{h}\right\|_{H^{1}} \leq C_{\varepsilon}^{G a l} h
$$

where

$$
C_{\varepsilon}^{G a l}=C_{I, \varepsilon}^{G a l}+C_{I I, \varepsilon}^{G a l}
$$

with

$$
C_{I, \varepsilon}^{G a l}=\frac{C}{\sqrt{\varepsilon}}, \quad C_{I I, \varepsilon}^{G a l}=C \sup _{x \in[0,1]} \frac{\exp \left(-x \sqrt{q^{*} / \varepsilon}\right)+\exp \left(-(1-x) \sqrt{q^{*} / \varepsilon}\right)}{\varepsilon \sqrt{\varepsilon}} .
$$

The part $C_{I, \varepsilon}^{G a l}$ is inherent to the singular perturbation problem itself whereas the part $C_{I I, \varepsilon}^{G a l}$ is due to the numerical method.

As is seen from the above estimate, the part $C_{I I, \varepsilon}^{G a l}$ is the main cause of worry. This part will keep on dominating since $\varepsilon \ll 1$. As a result, for the successful implementation of the finite element method, one will require that $h \leq \varepsilon \sqrt{\varepsilon}$, which is a severe restriction. To deal with this situation, we propose two new methods in the next two sections. 


\section{Singular Function Method}

This particular finite element method has augmented space of trial and test functions. In order to capture the singular nature of the solution $y(x)$ into the numerical method, we follow an idea which was first introduced by Strang and Fix [22] in the context of boundary value problems with corner singularities. More precisely, we replace the space $V_{h}$ by the augmented finite dimensional subspace of $H_{0}^{1}$ defined by

$$
V_{h}^{+}=V_{h} \oplus \operatorname{span}\left\{S_{0}, S_{1}\right\}
$$

The singular function method (SFM) reads as follows: find $y_{h}^{+} \in V_{h}^{+}$such that

$$
a\left(y_{h}^{+}, v_{h}^{+}\right)=\left(f, v_{h}^{+}\right) \quad \forall v_{h}^{+} \in V_{h}^{+} .
$$

The discrete problem (4.2) is well-posed due to the Lax-Milgram lemma.

We have the following result:

Theorem 4.1 The SFM has the asymptotic error estimate

$$
\left\|y-y_{h}^{+}\right\|_{H^{1}} \leq \frac{C}{\sqrt{\varepsilon}} h\left\|g^{\prime \prime}\right\|_{L^{2}} \equiv C_{I, \varepsilon}^{G a l} h
$$

where $g(x)$ is the regular part of $y(x)$ in Theorem 2.1 .

Proof. Since $V_{h}^{+}$is a subspace of $H_{0}^{1}(0,1)$, the analogue of (3.3) holds for the problem (4.2). Thus, we have

$$
\left\|y-y_{h}^{+}\right\|_{H^{1}} \leq \frac{C}{\sqrt{\varepsilon}} \inf _{v_{h}^{+} \in V_{h}^{+}}\left\|y-v_{h}^{+}\right\|_{H^{1}}
$$

Having the singular decomposition of $y(x)$ given in Theorem 2.1, it follows from (4.3) and from the choice

$$
v_{h}^{+}:=\gamma_{0} S_{0}+\gamma_{1} S_{1}+\Pi_{h} g \in V_{h}^{+}
$$

that

$$
\left\|y-y_{h}^{+}\right\|_{H^{1}} \leq \frac{C}{\sqrt{\varepsilon}}\left\|g-\Pi_{h} g\right\|_{H^{1}} .
$$

But the interpolation theory in Sobolev spaces and the regularity of $g(x)$ in Theorem 2.1 yield

$$
\left\|g-\Pi_{h} g\right\|_{H^{1}} \leq C h\left\|g^{\prime \prime}\right\|_{L^{2}} \leq C h\left\|g^{\prime \prime}\right\|_{C^{0}} .
$$

This combined with (4.4) completes the proof of the theorem.

Clearly, the space of trial and test functions $V_{h}^{+}$is advantageous over the standard space $V_{h}$ as it gives the above improved error estimates where we got rid of the part $C_{I I, \varepsilon}^{G a l}$ that arose from the standard FEM. However, the story is still not appealing in terms of getting the uniform convergence, because of the part $C_{I, \varepsilon}^{G a l}$. One way of looking into this aspect is to work with different norms, namely, the norm $\|\cdot\|_{L^{2}}$ and the energy norm $\|\cdot\|_{a}$.

The energy norm is inherent to the problem (1.3) and is given by

$$
\|v\|_{a}:=\sqrt{\int_{0}^{1}\left[\varepsilon p(x)\left(v^{\prime}(x)\right)^{2}+q(x)(v(x))^{2}\right] d x} .
$$

In this framework, we have the following $\varepsilon$-uniform convergence result: 
Theorem 4.2 The SFM is E-uniformly convergent of order one and two in the energy-norm and $L^{2}$-norm, respectively. That is

$$
\left\|y-y_{h}^{+}\right\|_{a} \leq C h
$$

and

$$
\left\|y-y_{h}^{+}\right\|_{L^{2}} \leq C h^{2}
$$

Proof. If we take $v=v^{+} \in V_{h}^{+}$in (1.3) and subtract the resulting equation from (4.2), we obtain

$$
a\left(y-y_{h}^{+}, v_{h}^{+}\right)=0
$$

Consequently,

$$
a\left(y-y_{h}^{+}, y-y_{h}^{+}\right)=\inf _{v^{+} \in V_{h}^{+}} a\left(y-y_{h}^{+}, y-v_{h}^{+}\right) .
$$

For $v_{h}^{+}=\gamma_{0} S_{0}+\gamma_{1} S_{1}+\Pi_{h} g$, we have then

$$
\begin{aligned}
\left\|y-y_{h}^{+}\right\|_{a}^{2} & \leq\left|a\left(y-y_{h}^{+}, y-v_{h}^{+}\right)\right| \\
& =\left|a\left(y-y_{h}^{+}, g-\Pi_{h} g\right)\right| \\
& \leq\left\|y-y_{h}^{+}\right\|_{a}\left\|g-\Pi_{h} g\right\|_{a} \quad \text { (by Cauchy-Schwarz inequality) } \\
& \leq C\left\|y-y_{h}^{+}\right\|_{a}\left\|g-\Pi_{h} g\right\|_{H^{1}} \\
& \leq C h\left\|y-y_{h}^{+}\right\|_{a}\left\|g^{\prime \prime}\right\|_{C^{0}}(\text { by }(4.5)) .
\end{aligned}
$$

Thus

$$
\left\|y-y_{h}^{+}\right\|_{a} \leq C h .
$$

On the other hand, with $\phi \in L^{2}$, we associate the functions $y(\phi) \in H_{0}^{1}$ and $y_{h}^{+}(\phi) \in V_{h}^{+}$ which are the unique solutions of the adjoint problems to (1.3) and (4.2):

$$
a(v, y(\phi))=(\phi, v) \quad \forall v \in H_{0}^{1}
$$

and

$$
a\left(v_{h}^{+}, y_{h}^{+}(\phi)\right)=\left(\phi, v_{h}^{+}\right) \quad \forall v_{h}^{+} \in V_{h}^{+} .
$$

Denote by $g(\phi)$ the regular part of $y(\phi)$. We now proceed by the Aubin-Nitsche's duality argument [3] as follows:

$$
\begin{aligned}
\left\|y-y_{h}^{+}\right\|_{L^{2}} & =\sup _{0 \neq \phi \in L^{2}} \frac{\left|\left(y-y_{h}^{+}, \phi\right)\right|}{\|\phi\|_{L^{2}}} \\
& \left.=\sup _{0 \neq \phi \in L^{2}} \frac{\left|a\left(y(\phi), y-y_{h}^{+}\right)\right|}{\|\phi\|_{L^{2}}} \text { (by }(4.7)\right) \\
& =\sup _{0 \neq \phi \in L^{2}} \frac{a\left(y(\phi)-y_{h}^{+}(\phi), y-y_{h}^{+}\right)}{\|\phi\|_{L^{2}}} \text { since } a\left(y_{h}^{+}(\phi), y-y_{h}^{+}\right)=0 \\
& \leq \sup _{0 \neq \phi \in L^{2}} \frac{\left\|y(\phi)-y_{h}^{+}(\phi)\right\|_{a}\left\|y-y_{h}^{+}\right\|_{a}}{\|\phi\|_{L^{2}}} \\
& \leq \sup _{0 \neq \phi \in L^{2}} \frac{C h\left\|g^{\prime \prime}(\phi)\right\|_{C^{0}} h\left\|g^{\prime \prime}\right\|_{C^{0}}}{\|\phi\|_{L^{2}}} \quad(\text { by } \quad(4.5)) \\
& \leq C h^{2}\left\|g^{\prime \prime}\right\|_{C^{0}} \sup _{0 \neq \phi \in L^{2}} \frac{\left\|g^{\prime \prime}(\phi)\right\|_{C^{0}}}{\|\phi\|_{L^{2}}} \\
& \leq C h^{2} .
\end{aligned}
$$

The last inequality results from the fact that the adjoint problem is regular. 


\section{Mesh Refinement Method}

We start with the standard FEM for Eq. (1.3), as defined in Eq. (3.2). We will make the necessary modifications shortly. In the energy norm, Céa's lemma, i.e. (3.3), reads as

$$
\left\|y-y_{h}\right\|_{a}^{2}=\inf _{v_{h} \in V_{h}}\left\|y-v_{h}\right\|_{a}^{2}
$$

This yields the series of relations

$$
\begin{aligned}
\left\|y-y_{h}\right\|_{a}^{2} & \leq\left\|y-\Pi_{h} y\right\|_{a}^{2} \\
& \leq C \sum_{i=0}^{n-1}\left(\varepsilon\left|y-\Pi_{i} y\right|_{H^{1}\left(x_{i}, x_{i+1}\right)}^{2}+\left\|y-\Pi_{i} y\right\|_{L^{2}\left(x_{i}, x_{i+1}\right)}^{2}\right)
\end{aligned}
$$

where we recall that $\Pi_{h}$ is the $V_{h}$-global interpolation operator, whereas $\Pi_{i}$ is the local interpolation operator on the space of polynomials of degree $\leq 1$ restricted to the interval $\left[x_{i}, x_{i+1}\right]$. Thus, estimating the error $\left\|y-y_{h}\right\|_{a}^{2}$ is reduced to estimating each local interpolation error $\varepsilon\left|y-\Pi_{i} y\right|_{H^{1}\left(x_{i}, x_{i+1}\right)}^{2}$ and $\left\|y-\Pi_{i} y\right\|_{L^{2}\left(x_{i}, x_{i+1}\right)}^{2}$. It is convenient to deal with the end point $x_{0}=0$, the situation of the node $x_{n}=1$ being dealt with by symmetry. We consider the following two cases:

First case: $i \neq 0$. We are far away from the layer region $\left[0, x_{1}\right]$. We use the decomposition in Theorem 2.1 (in which we ignore $\gamma_{1} S_{1}$ as we work with the end point 0 ). This yields

$$
\left\|y-\Pi_{i} y\right\|_{L^{2}\left(x_{i}, x_{i+1}\right)}^{2} \leq C\left[\left\|g-\Pi_{i} g\right\|_{L^{2}\left(x_{i}, x_{i+1}\right)}^{2}+\left\|S_{0}-\Pi_{i} S_{0}\right\|_{L^{2}\left(x_{i}, x_{i+1}\right)}^{2}\right]
$$

and

$$
\varepsilon\left|y-\Pi_{i} y\right|_{H^{1}\left(x_{i}, x_{i+1}\right)}^{2} \leq C\left[\left|g-\Pi_{i} g\right|_{H^{1}\left(x_{i}, x_{i+1}\right)}^{2}+\varepsilon\left|S_{0}-\Pi_{i} S_{0}\right|_{H^{1}\left(x_{i}, x_{i+1}\right)}^{2}\right] .
$$

For the purpose of obtaining suitable interpolation estimates, it is essential not to get rid in (5.3) of $\varepsilon$ in front of the semi-norm related to the singular function $S_{0}$. By interpolation theory in Sobolev spaces applied to $g-\Pi_{i} g$ and to $S_{0}-\Pi_{i} S_{0}$, we have the estimates (see [3])

$$
\begin{gathered}
\left(x_{i+1}-x_{i}\right)^{-2}\left\|g-\prod_{i} g\right\|_{L^{2}\left(x_{i}, x_{i+1}\right)}^{2}+\varepsilon\left|g-\Pi_{i} g\right|_{H^{1}\left(x_{i}, x_{i+1}\right)}^{2} \leq C\left(x_{i+1}-x_{i}\right)^{2}\left\|g^{\prime \prime}\right\|_{L^{2}}^{2}, \\
\left\|S_{0}-\Pi_{i} S_{0}\right\|_{L^{2}\left(x_{i}, x_{i+1}\right)}^{2} \leq C\left(x_{i+1}-x_{i}\right)^{4}\left\|S_{0}^{\prime \prime}\right\|_{L^{2}\left(x_{i}, x_{i+1}\right)}^{2}
\end{gathered}
$$

and

$$
\varepsilon\left|S_{0}-\Pi_{i} S_{0}\right|_{H^{1}\left(x_{i}, x_{i+1}\right)}^{2} \leq C \varepsilon\left(x_{i+1}-x_{i}\right)^{2} \|\left. S_{0}^{\prime \prime}\right|_{L^{2}\left(x_{i}, x_{i+1}\right)} ^{2} .
$$

The expression of the singular function in Theorem 2.1 permits to explicitly compute the derivative:

$$
S_{0}^{\prime \prime}(x)=\varepsilon^{-1} q(0) \exp \left(-x \sqrt{\varepsilon^{-1} q(0)}\right) .
$$

Thus

$$
\begin{aligned}
\left\|S_{0}^{\prime \prime}\right\|_{L^{2}\left(x_{i}, x_{i+1}\right)}^{2}= & 2^{-1}\left(\varepsilon^{-1} q(0)\right)^{3 / 2}\left[\exp \left(-2 x_{i} \sqrt{\varepsilon^{-1} q(0)}\right)-\right. \\
& \left.\exp \left(-2 x_{i+1} \sqrt{\varepsilon^{-1} q(0)}\right)\right] \\
\leq & 2^{-1}\left(\varepsilon^{-1} q(0)\right)^{3 / 2} \exp \left(-2 x_{i} \sqrt{\varepsilon^{-1} q(0)}\right) \\
\leq & C x_{i}^{-3} \text { as } \frac{u^{3}}{3 !} \exp \left(-u^{3}\right) \leq 1 \text { with } u=2 x_{i} \sqrt{\varepsilon^{-1} q(0)} .
\end{aligned}
$$


Consequently, we end up with the following estimates for the solution $y$ :

$$
\left\|y-\Pi_{i} y\right\|_{L^{2}\left(x_{i}, x_{i+1}\right)}^{2} \leq C\left(x_{i+1}-x_{i}\right)^{4}\left[\left\|g^{\prime \prime}\right\|_{L^{2}}^{2}+x_{i}^{-3}\right]
$$

and

$$
\varepsilon\left|y-\Pi_{i} y\right|_{H^{1}\left(x_{i}, x_{i+1}\right)}^{2} \leq C\left(x_{i+1}-x_{i}\right)^{2}\left[\left\|g^{\prime \prime}\right\|_{L^{2}}^{2}+\varepsilon x_{i}^{-3}\right] .
$$

Second case: $i=0$. We are in the layer region $\left[0, x_{1}\right]$. If we write the analogues of (5.2) and (5.3) and proceed as in the first case above, interpolation theory gives the following counterparts of the estimates (5.8) and (5.9):

$$
\left\|y-\Pi_{0} y\right\|_{L^{2}\left(0, x_{1}\right)}^{2} \leq C x_{1}^{4}\left[\left\|g^{\prime \prime}\right\|_{L^{2}}^{2}+\varepsilon^{-3 / 2}\right]
$$

and

$$
\varepsilon\left|y-\Pi_{0} y\right|_{H^{1}\left(0, x_{1}\right)}^{2} \leq C x_{1}^{2}\left[\|\left. g^{\prime \prime}\right|_{L^{2}} ^{2}+\varepsilon^{-1 / 2}\right] .
$$

The announced modification of the standard FEM is the Mesh Refinement Method (MRM) obtained as follows. On the nodes $\left(x_{i}\right)$, we impose the mesh refinement condition

$$
\left(x_{i+1}-x_{i}\right)^{2} \varepsilon x_{i}^{-3} \leq C h^{2}
$$

for $i \neq 0$, whereas we require

$$
x_{1}^{2} \varepsilon^{-3 / 2} \leq C h^{2}
$$

whenever $i=0$.

Remark 5.1 The analysis done in the two cases above show that, for the MRM, we could work with the decomposition (2.1) in which the singular functions do not take the values zero at the end-points of the interval.

Theorem 5.2 If the finite element space $V_{h}$ is constructed according to the mesh refinement conditions (5.12) and (5.13), then the FEM (3.2) is $\varepsilon$-uniformly convergent of order one and two in the energy and $L^{2}$-norms, respectively:

$$
\left\|y-y_{h}\right\|_{a} \leq C h
$$

and

$$
\left\|y-y_{h}\right\|_{L^{2}} \leq C h^{2}
$$

A typical example that meets the mesh refinement requirements (5.12) and (5.13) consists of the nodes defined by

$$
x_{i}=\left(\frac{i}{n}\right)^{\nu}
$$

where the parameter $\nu>1$ is chosen such that it is less than or equal to the optimal value

$$
\nu=1-\frac{\log \left(C \varepsilon^{3 / 2}\right)}{2 \log n} .
$$

Eq. (5.15) is readily derived by using (5.14) in (5.13).

Remark 5.3 We come back to the initial situation where both end-points $x=0$ and $x=1$ of the interval $[0,1]$ must be incorporated. We assume that $n$ is an even number: $n=2 N$. By symmetry, we obtain from (5.14) the refined mesh given by the nodes

$$
x_{i}:=\left\{\begin{array}{l}
\frac{1}{2}\left(\frac{i}{N}\right)^{\nu} \quad \text { if } 0 \leq i \leq N \\
1-\frac{1}{2}\left(\frac{2 N-i}{N}\right)^{\nu} \text { if } N \leq i \leq 2 N
\end{array}\right.
$$

for $\nu>1$ as above. 
Table 1: Values of $\nu$ obtained using (5.15) with $C=1$

\begin{tabular}{|c|ccccc|}
\hline$\varepsilon$ & $n=100$ & $n=200$ & $n=400$ & $n=800$ & $n=1600$ \\
\hline 1.0 & 1.0000 & 1.0000 & 1.0000 & 1.0000 & 1.0000 \\
$10^{-1}$ & 1.4414 & 1.3750 & 1.3259 & 1.2882 & 1.2583 \\
$10^{-2}$ & 1.8829 & 1.7500 & 1.6519 & 1.5765 & 1.5167 \\
$10^{-3}$ & 2.3243 & 2.1250 & 1.9778 & 1.8647 & 1.7750 \\
$10^{-4}$ & 2.7658 & 2.5000 & 2.3038 & 2.1529 & 2.0334 \\
$10^{-5}$ & 3.2072 & 2.8750 & 2.6297 & 2.4412 & 2.2917 \\
$10^{-6}$ & 3.6487 & 3.2500 & 2.9556 & 2.7294 & 2.5501 \\
$10^{-7}$ & 4.0901 & 3.6250 & 3.2816 & 3.0176 & 2.8084 \\
$10^{-8}$ & 4.5316 & 4.0000 & 3.6075 & 3.3059 & 3.0668 \\
$10^{-9}$ & 4.9730 & 4.3750 & 3.9335 & 3.5941 & 3.3251 \\
\hline
\end{tabular}

Remark 5.4 To keep the method self-adaptive, it is good to choose the $\nu$ according to the formula (5.15). As is shown in Table 1, the values of $\nu$ are ranging from 1 to 4.973. Thus, for a fixed $n$, the values of $\nu$ given by (5.15) increase for decreasing $\varepsilon$, whereas the lower bound is always 1 ( $\nu=1$ gives the uniform mesh throughout the region). However, our numerical simulations show that $\varepsilon$-uniform convergence is achieved with a fixed value of $\nu$.

\section{Numerical Implementation}

A basis for $V_{h}^{+}$is $\left\{\phi_{1}^{+}, \phi_{2}^{+}, \ldots, \phi_{n}^{+}, \phi_{n+1}^{+}\right\}$where $\phi_{j}^{+}, j=1(1) n-1$, are the usual continuous piecewise linear basis functions whereas $\phi_{n}^{+}=S_{0}$ and $\phi_{n+1}^{+}=S_{1}$.

Setting

$$
y_{h}^{+}=\sum_{j=1}^{n+1} \alpha_{j}^{+} \phi_{j}^{+}
$$

the SFM is equivalent to solving the $(n+1) \times(n+1)$ system

$$
\mathbf{K}^{+} \boldsymbol{\alpha}^{+}=\boldsymbol{\beta}^{+}
$$

with

$$
\mathbf{K}^{+}=\left[\begin{array}{ll}
\mathbf{K}_{11} & \mathbf{K}_{12} \\
\mathbf{K}_{21} & \mathbf{K}_{22}
\end{array}\right], \boldsymbol{\alpha}^{+}=\left(\begin{array}{c}
\boldsymbol{\alpha}_{1}^{+} \\
\alpha_{n} \\
\alpha_{n+1}
\end{array}\right), \boldsymbol{\beta}^{+}=\left(\begin{array}{c}
\boldsymbol{\beta}_{1}^{+} \\
\beta_{n} \\
\beta_{n+1}
\end{array}\right) .
$$

Note that the $(n-1) \times(n-1)$ system

$$
\mathbf{K}_{11} \boldsymbol{\alpha}_{1}^{+}=\boldsymbol{\beta}_{1}^{+}
$$

corresponds to the standard FEM part, whereas the other matrices $\mathbf{K}_{21}^{T}=\left(\mathbf{K}_{12}\right)_{n-1,2}$ and $\left(\mathbf{K}_{22}\right)_{2,2}$ are contributed by the singular functions. Although the SFM has a uniform mesh like the standard FEM, the said-additional contribution of the singular functions destroys the tridiagonal structure of the stiffness matrix. As a result, special attention is required for the numerical solution of the system (6.1). (See, for example, [5] for possible techniques). On the contrary, the algebraic structure of the system involved in the MRM does not change: the stiffness matrix is tridiagonal, though the mesh is not uniform. 
Numerical results corresponding to problem (1.1) are tabulated for the three methods FEM, SFM and MRM with

$$
p(x) \equiv 1, q(x) \equiv 1 \text { and } f(x)=1+2 \sqrt{\varepsilon}\left[e^{-x / \sqrt{\varepsilon}}+e^{-(1-x) / \sqrt{\varepsilon}}\right] .
$$

The exact solution for the corresponding problem is given by

$$
y(x)=1+(x-1) e^{-x / \sqrt{\varepsilon}}-x e^{-(1-x) / \sqrt{\varepsilon}} .
$$

In the case of the SFM, the following further aspect is of interest with regard to the simulation of $\varepsilon$-uniform convergence. The computations corresponding to the matrix entries $\mathbf{K}_{12}, \mathbf{K}_{21}$ and $\mathbf{K}_{22}$ involve singular functions $S_{0}$ and $S_{1}$. The $k$-th order derivatives of this functions satisfy (cf. (2.2):

$$
S_{J}^{(k)}=O\left(\varepsilon^{-k / 2}\right), \quad J=1,2 .
$$

This means that the numerical integration formulae, used to evaluate the local stiffness matrices for these parts, have unacceptable remainder terms when $\varepsilon \rightarrow 0$. This pollutes the $\varepsilon$-uniform behavior of the numerical solutions in the error tables (see, Tables 3 and 7 ), even though theoretically we have $\varepsilon$-uniform convergence. However the SFM behaves better than the FEM as shown by the numerical results under the severe condition $h \leq \varepsilon$, which is needed for the FEM.

Results are tabulated for the errors in the $L^{2}$ and energy norms for the three methods.

\section{Conclusions and Future Directions}

We have designed two appropriate finite element methods (the SFM and the MRM) for the numerical solution of singular perturbation problems. Both methods use the intrinsic singular feature of the solution. In the SFM, the standard space of trial and test functions (piecewise linear) is augmented by the space spanned by the singular functions on a uniform mesh, while the MRM still uses the space of piecewise linear functions but on a refined mesh. The two methods theoretically provide $\varepsilon$-uniform convergence of second and first order in the $L^{2}$ and energy-norms, respectively. Numerically, we find the results consistent with the theory in the case of MRM. Likewise, this happens to the SFM provided that the numerical integration errors on the entries of the stiffness matrix and of the load vector involving the singular functions are taken into consideration.

It should be noted that we have extended the SFM and the MRF, known for boundary value problems with corner singularities, to singular perturbation problems. Our approach is innovative in that it has never been considered in the literature for the problems studied here and that the nature of singularities of SPPs is completely different from those of problems with corners.

For convenience, we compare our mesh generation strategy with the widely used piecewise uniform meshes of Shishkin (see, e.g., [17]). Numerical results are presented in Tables 5 and 9. The superiority of our results is due to the fact that the mesh does not have unwanted grid points like the Shishkin mesh.

Our future research plan includes extending this study to two-dimensional singular perturbation problems on rough domains such as polygons. The rough geometry of the domain, which is the setting in which the MRM and the SFM were initially developed in [2] and [22], brings into the problem an additional difficulty of having non-smooth solutions. The $h p$ version of the finite element method for such problems is analyzed in [23]. 
Table 2: Standard FEM: Errors in $L^{2}$-norm

\begin{tabular}{|c|ccccc|}
\hline$\varepsilon$ & $n=100$ & $n=200$ & $n=400$ & $n=800$ & $n=1600$ \\
\hline 1.0 & $3.0917 \mathrm{e}-06$ & $7.7291 \mathrm{e}-07$ & $1.9323 \mathrm{e}-07$ & $4.8306 \mathrm{e}-08$ & $1.2069 \mathrm{e}-08$ \\
$10^{-2}$ & $1.4327 \mathrm{e}-04$ & $3.5804 \mathrm{e}-05$ & $8.9498 \mathrm{e}-06$ & $2.2374 \mathrm{e}-06$ & $5.5934 \mathrm{e}-07$ \\
$10^{-3}$ & $7.6028 \mathrm{e}-04$ & $1.8985 \mathrm{e}-04$ & $4.7430 \mathrm{e}-05$ & $1.1854 \mathrm{e}-05$ & $2.9633 \mathrm{e}-06$ \\
$10^{-4}$ & $4.2260 \mathrm{e}-03$ & $1.0524 \mathrm{e}-03$ & $2.6284 \mathrm{e}-04$ & $6.5643 \mathrm{e}-05$ & $1.6402 \mathrm{e}-05$ \\
$10^{-5}$ & $2.1910 \mathrm{e}-02$ & $5.9533 \mathrm{e}-03$ & $1.4741 \mathrm{e}-03$ & $3.6789 \mathrm{e}-04$ & $9.1872 \mathrm{e}-05$ \\
$10^{-6}$ & $4.5701 \mathrm{e}-02$ & $2.3853 \mathrm{e}-02$ & $8.1432 \mathrm{e}-03$ & $2.0817 \mathrm{e}-03$ & $5.1671 \mathrm{e}-04$ \\
$10^{-7}$ & $5.0564 \mathrm{e}-02$ & $3.4563 \mathrm{e}-02$ & $2.1394 \mathrm{e}-02$ & $9.7987 \mathrm{e}-03$ & $2.9256 \mathrm{e}-03$ \\
$10^{-8}$ & $5.1083 \mathrm{e}-02$ & $3.5998 \mathrm{e}-02$ & $2.5111 \mathrm{e}-02$ & $1.6821 \mathrm{e}-02$ & $9.6736 \mathrm{e}-03$ \\
$10^{-9}$ & $5.1135 \mathrm{e}-02$ & $3.6145 \mathrm{e}-02$ & $2.5524 \mathrm{e}-02$ & $1.7950 \mathrm{e}-02$ & $1.2420 \mathrm{e}-02$ \\
\hline
\end{tabular}

Table 3: SFM: Errors in $L^{2}$-norm

\begin{tabular}{|c|ccccc|}
\hline$\varepsilon$ & $n=100$ & $n=200$ & $n=400$ & $n=800$ & $n=1600$ \\
\hline 1.0 & $4.5537 \mathrm{e}-08$ & $1.1468 \mathrm{e}-08$ & $2.8721 \mathrm{e}-09$ & $7.1834 \mathrm{e}-10$ & $1.7950 \mathrm{e}-10$ \\
$10^{-2}$ & $9.8697 \mathrm{e}-06$ & $2.9408 \mathrm{e}-06$ & $7.6706 \mathrm{e}-07$ & $1.9379 \mathrm{e}-07$ & $4.8574 \mathrm{e}-08$ \\
$10^{-3}$ & $1.1035 \mathrm{e}-04$ & $5.4920 \mathrm{e}-06$ & $8.0440 \mathrm{e}-07$ & $2.8469 \mathrm{e}-07$ & $7.7205 \mathrm{e}-08$ \\
$10^{-4}$ & $2.5321 \mathrm{e}-03$ & $3.2538 \mathrm{e}-04$ & $2.6746 \mathrm{e}-05$ & $1.5162 \mathrm{e}-06$ & $7.0652 \mathrm{e}-08$ \\
$10^{-5}$ & $1.9747 \mathrm{e}-02$ & $4.4155 \mathrm{e}-03$ & $7.4312 \mathrm{e}-04$ & $8.2585 \mathrm{e}-05$ & $6.2894 \mathrm{e}-06$ \\
$10^{-6}$ & $5.4347 \mathrm{e}-02$ & $2.4582 \mathrm{e}-02$ & $6.8904 \mathrm{e}-03$ & $1.4030 \mathrm{e}-03$ & $2.0868 \mathrm{e}-04$ \\
$10^{-7}$ & $5.9906 \mathrm{e}-02$ & $4.1523 \mathrm{e}-02$ & $2.4721 \mathrm{e}-02$ & $9.4021 \mathrm{e}-03$ & $2.3231 \mathrm{e}-03$ \\
$10^{-8}$ & $5.9530 \mathrm{e}-02$ & $4.2298 \mathrm{e}-02$ & $2.9900 \mathrm{e}-02$ & $2.0204 \mathrm{e}-02$ & $1.0622 \mathrm{e}-02$ \\
$10^{-9}$ & $5.9223 \mathrm{e}-02$ & $4.1985 \mathrm{e}-02$ & $2.9812 \mathrm{e}-02$ & $2.1175 \mathrm{e}-02$ & $1.4864 \mathrm{e}-02$ \\
\hline
\end{tabular}

Table 4: MRM: Errors in $L^{2}$-norm $(\nu=4)$

\begin{tabular}{|c|ccccc|}
\hline$\varepsilon$ & $n=100$ & $n=200$ & $n=400$ & $n=800$ & $n=1600$ \\
\hline 1.0 & $3.0023 \mathrm{e}-05$ & $7.8687 \mathrm{e}-06$ & $2.0120 \mathrm{e}-06$ & $5.0854 \mathrm{e}-07$ & $1.2782 \mathrm{e}-07$ \\
$10^{-2}$ & $2.9317 \mathrm{e}-04$ & $7.4092 \mathrm{e}-05$ & $1.8622 \mathrm{e}-05$ & $4.6678 \mathrm{e}-06$ & $1.1685 \mathrm{e}-06$ \\
$10^{-3}$ & $2.5619 \mathrm{e}-04$ & $6.4026 \mathrm{e}-05$ & $1.6005 \mathrm{e}-05$ & $4.0012 \mathrm{e}-06$ & $1.0003 \mathrm{e}-06$ \\
$10^{-4}$ & $2.4781 \mathrm{e}-04$ & $6.1915 \mathrm{e}-05$ & $1.5476 \mathrm{e}-05$ & $3.8690 \mathrm{e}-06$ & $9.6723 \mathrm{e}-07$ \\
$10^{-5}$ & $2.4530 \mathrm{e}-04$ & $6.1259 \mathrm{e}-05$ & $1.5311 \mathrm{e}-05$ & $3.8274 \mathrm{e}-06$ & $9.5683 \mathrm{e}-07$ \\
$10^{-6}$ & $2.4473 \mathrm{e}-04$ & $6.1066 \mathrm{e}-05$ & $1.5259 \mathrm{e}-05$ & $3.8143 \mathrm{e}-06$ & $9.5355 \mathrm{e}-07$ \\
$10^{-7}$ & $2.4490 \mathrm{e}-04$ & $6.1030 \mathrm{e}-05$ & $1.5244 \mathrm{e}-05$ & $3.8103 \mathrm{e}-06$ & $9.5252 \mathrm{e}-07$ \\
$10^{-8}$ & $2.4551 \mathrm{e}-04$ & $6.1062 \mathrm{e}-05$ & $1.5243 \mathrm{e}-05$ & $3.8092 \mathrm{e}-06$ & $9.5221 \mathrm{e}-07$ \\
$10^{-9}$ & $2.4634 \mathrm{e}-04$ & $6.1146 \mathrm{e}-05$ & $1.5247 \mathrm{e}-05$ & $3.8092 \mathrm{e}-06$ & $9.5213 \mathrm{e}-07$ \\
\hline
\end{tabular}

Table 5: Errors in $L^{2}$-norm (when Shishkin Mesh is used)

\begin{tabular}{|c|ccccc|}
\hline$\varepsilon$ & $n=100$ & $n=200$ & $n=400$ & $n=800$ & $n=1600$ \\
\hline 1.0 & $5.3383 \mathrm{e}-05$ & $1.3435 \mathrm{e}-05$ & $3.3698 \mathrm{e}-06$ & $8.4386 \mathrm{e}-07$ & $2.1114 \mathrm{e}-07$ \\
$10^{-2}$ & $4.8705 \mathrm{e}-04$ & $1.2961 \mathrm{e}-04$ & $3.3454 \mathrm{e}-05$ & $8.4999 \mathrm{e}-06$ & $2.1423 \mathrm{e}-06$ \\
$10^{-3}$ & $8.5324 \mathrm{e}-04$ & $2.8435 \mathrm{e}-04$ & $9.3449 \mathrm{e}-05$ & $2.9786 \mathrm{e}-05$ & $9.2214 \mathrm{e}-06$ \\
$10^{-4}$ & $1.2797 \mathrm{e}-03$ & $3.6176 \mathrm{e}-04$ & $9.7827 \mathrm{e}-05$ & $2.6315 \mathrm{e}-05$ & $7.1159 \mathrm{e}-06$ \\
$10^{-5}$ & $1.3870 \mathrm{e}-03$ & $5.2455 \mathrm{e}-04$ & $1.6275 \mathrm{e}-04$ & $4.4786 \mathrm{e}-05$ & $1.1807 \mathrm{e}-05$ \\
$10^{-6}$ & $1.1842 \mathrm{e}-03$ & $4.7575 \mathrm{e}-04$ & $1.8909 \mathrm{e}-04$ & $6.6336 \mathrm{e}-05$ & $1.9601 \mathrm{e}-05$ \\
$10^{-7}$ & $1.1579 \mathrm{e}-03$ & $4.4004 \mathrm{e}-04$ & $1.6557 \mathrm{e}-04$ & $6.4155 \mathrm{e}-05$ & $2.4471 \mathrm{e}-05$ \\
$10^{-8}$ & $1.1676 \mathrm{e}-03$ & $4.4324 \mathrm{e}-04$ & $1.6252 \mathrm{e}-04$ & $5.8801 \mathrm{e}-05$ & $2.1745 \mathrm{e}-05$ \\
$10^{-9}$ & $1.1731 \mathrm{e}-03$ & $4.4728 \mathrm{e}-04$ & $1.6471 \mathrm{e}-04$ & $5.9289 \mathrm{e}-05$ & $2.1073 \mathrm{e}-05$ \\
\hline
\end{tabular}


Table 6: Standard FEM: Errors in Energy-norm

\begin{tabular}{|c|ccccc|}
\hline$\varepsilon$ & $n=100$ & $n=200$ & $n=400$ & $n=800$ & $n=1600$ \\
\hline 1.0 & $3.6095 \mathrm{e}-02$ & $1.8166 \mathrm{e}-02$ & $9.1127 \mathrm{e}-03$ & $4.5638 \mathrm{e}-03$ & $2.2838 \mathrm{e}-03$ \\
$10^{-2}$ & $3.6600 \mathrm{e}-02$ & $1.9287 \mathrm{e}-02$ & $9.9045 \mathrm{e}-03$ & $5.0194 \mathrm{e}-03$ & $2.5267 \mathrm{e}-03$ \\
$10^{-3}$ & $4.8585 \mathrm{e}-02$ & $2.8136 \mathrm{e}-02$ & $1.5199 \mathrm{e}-02$ & $7.9070 \mathrm{e}-03$ & $4.0337 \mathrm{e}-03$ \\
$10^{-4}$ & $4.9915 \mathrm{e}-02$ & $3.6024 \mathrm{e}-02$ & $2.2334 \mathrm{e}-02$ & $1.2545 \mathrm{e}-02$ & $6.6645 \mathrm{e}-03$ \\
$10^{-5}$ & $3.6404 \mathrm{e}-02$ & $3.1576 \mathrm{e}-02$ & $2.5473 \mathrm{e}-02$ & $1.7340 \mathrm{e}-02$ & $1.0324 \mathrm{e}-02$ \\
$10^{-6}$ & $3.7455 \mathrm{e}-02$ & $2.4831 \mathrm{e}-02$ & $1.9318 \mathrm{e}-02$ & $1.6780 \mathrm{e}-02$ & $1.2779 \mathrm{e}-02$ \\
$10^{-7}$ & $3.9116 \mathrm{e}-02$ & $2.7229 \mathrm{e}-02$ & $1.8311 \mathrm{e}-02$ & $1.2453 \mathrm{e}-02$ & $1.0396 \mathrm{e}-02$ \\
$10^{-8}$ & $3.9310 \mathrm{e}-02$ & $2.7750 \mathrm{e}-02$ & $1.9495 \mathrm{e}-02$ & $1.3456 \mathrm{e}-02$ & $8.9291 \mathrm{e}-03$ \\
$10^{-9}$ & $3.9330 \mathrm{e}-02$ & $2.7806 \mathrm{e}-02$ & $1.9649 \mathrm{e}-02$ & $1.3857 \mathrm{e}-02$ & $9.6981 \mathrm{e}-03$ \\
\hline
\end{tabular}

Table 7: SFM: Errors in Energy-norm

\begin{tabular}{|c|ccccc|}
\hline$\varepsilon$ & $n=100$ & $n=200$ & $n=400$ & $n=800$ & $n=1600$ \\
\hline 1.0 & $4.2759 \mathrm{e}-04$ & $2.1913 \mathrm{e}-04$ & $1.1093 \mathrm{e}-04$ & $5.5810 \mathrm{e}-05$ & $2.7992 \mathrm{e}-05$ \\
$10^{-2}$ & $7.5812 \mathrm{e}-04$ & $3.8456 \mathrm{e}-04$ & $1.9704 \mathrm{e}-04$ & $9.9727 \mathrm{e}-05$ & $5.0164 \mathrm{e}-05$ \\
$10^{-3}$ & $3.3454 \mathrm{e}-03$ & $5.7559 \mathrm{e}-04$ & $1.2364 \mathrm{e}-04$ & $5.3861 \mathrm{e}-05$ & $2.7243 \mathrm{e}-05$ \\
$10^{-4}$ & $1.1153 \mathrm{e}-02$ & $4.7763 \mathrm{e}-03$ & $1.0538 \mathrm{e}-03$ & $1.6416 \mathrm{e}-04$ & $2.5805 \mathrm{e}-05$ \\
$10^{-5}$ & $1.6700 \mathrm{e}-02$ & $7.6935 \mathrm{e}-03$ & $5.1052 \mathrm{e}-03$ & $1.7010 \mathrm{e}-03$ & $3.2328 \mathrm{e}-04$ \\
$10^{-6}$ & $4.2175 \mathrm{e}-02$ & $1.9550 \mathrm{e}-02$ & $6.4596 \mathrm{e}-03$ & $3.9524 \mathrm{e}-03$ & $2.1434 \mathrm{e}-03$ \\
$10^{-7}$ & $4.6117 \mathrm{e}-02$ & $3.2054 \mathrm{e}-02$ & $1.9281 \mathrm{e}-02$ & $7.6381 \mathrm{e}-03$ & $2.7716 \mathrm{e}-03$ \\
$10^{-8}$ & $4.5788 \mathrm{e}-02$ & $3.2543 \mathrm{e}-02$ & $2.3031 \mathrm{e}-02$ & $1.5630 \mathrm{e}-02$ & $8.3495 \mathrm{e}-03$ \\
$10^{-9}$ & $4.5549 \mathrm{e}-02$ & $3.2292 \mathrm{e}-02$ & $2.2932 \mathrm{e}-02$ & $1.6295 \mathrm{e}-02$ & $1.1460 \mathrm{e}-02$ \\
\hline
\end{tabular}

Table 8: MRM: Errors in Energy-norm $(\nu=2)$

\begin{tabular}{|c|ccccc|}
\hline$\varepsilon$ & $n=100$ & $n=200$ & $n=400$ & $n=800$ & $n=1600$ \\
\hline 1.0 & $7.0030 \mathrm{e}-02$ & $3.7828 \mathrm{e}-02$ & $2.0161 \mathrm{e}-02$ & $1.0653 \mathrm{e}-02$ & $5.5943 \mathrm{e}-03$ \\
$10^{-2}$ & $3.9317 \mathrm{e}-02$ & $2.1417 \mathrm{e}-02$ & $1.1579 \mathrm{e}-02$ & $6.2096 \mathrm{e}-03$ & $3.3052 \mathrm{e}-03$ \\
$10^{-3}$ & $3.4342 \mathrm{e}-02$ & $1.8768 \mathrm{e}-02$ & $1.0209 \mathrm{e}-02$ & $5.5107 \mathrm{e}-03$ & $2.9505 \mathrm{e}-03$ \\
$10^{-4}$ & $3.1355 \mathrm{e}-02$ & $1.7089 \mathrm{e}-02$ & $9.3352 \mathrm{e}-03$ & $5.0716 \mathrm{e}-03$ & $2.7333 \mathrm{e}-03$ \\
$10^{-5}$ & $2.9340 \mathrm{e}-02$ & $1.5796 \mathrm{e}-02$ & $8.6198 \mathrm{e}-03$ & $4.7060 \mathrm{e}-03$ & $2.5533 \mathrm{e}-03$ \\
$10^{-6}$ & $2.7965 \mathrm{e}-02$ & $1.4802 \mathrm{e}-02$ & $7.9955 \mathrm{e}-03$ & $4.3668 \mathrm{e}-03$ & $2.3823 \mathrm{e}-03$ \\
$10^{-7}$ & $2.6369 \mathrm{e}-02$ & $1.4097 \mathrm{e}-02$ & $7.4832 \mathrm{e}-03$ & $4.0540 \mathrm{e}-03$ & $2.2152 \mathrm{e}-03$ \\
$10^{-8}$ & $2.4356 \mathrm{e}-02$ & $1.3393 \mathrm{e}-02$ & $7.1098 \mathrm{e}-03$ & $3.7873 \mathrm{e}-03$ & $2.0569 \mathrm{e}-03$ \\
$10^{-9}$ & $2.4470 \mathrm{e}-02$ & $1.2275 \mathrm{e}-02$ & $6.7849 \mathrm{e}-03$ & $3.5876 \mathrm{e}-03$ & $1.9182 \mathrm{e}-03$ \\
\hline
\end{tabular}

Table 9: Errors in Energy-norm (when Shishkin Mesh is used)

\begin{tabular}{|c|ccccc|}
\hline$\varepsilon$ & $n=100$ & $n=200$ & $n=400$ & $n=800$ & $n=1600$ \\
\hline 1.0 & $3.6095 \mathrm{e}-02$ & $1.8166 \mathrm{e}-02$ & $9.1127 \mathrm{e}-03$ & $4.5638 \mathrm{e}-03$ & $2.2838 \mathrm{e}-03$ \\
$10^{-2}$ & $3.6696 \mathrm{e}-02$ & $1.9300 \mathrm{e}-02$ & $9.9061 \mathrm{e}-03$ & $5.0196 \mathrm{e}-03$ & $2.5268 \mathrm{e}-03$ \\
$10^{-3}$ & $3.2241 \mathrm{e}-02$ & $1.9890 \mathrm{e}-02$ & $1.1751 \mathrm{e}-02$ & $6.7289 \mathrm{e}-03$ & $3.7697 \mathrm{e}-03$ \\
$10^{-4}$ & $1.7735 \mathrm{e}-02$ & $1.0882 \mathrm{e}-02$ & $6.4150 \mathrm{e}-03$ & $3.6698 \mathrm{e}-03$ & $2.0550 \mathrm{e}-03$ \\
$10^{-5}$ & $1.0047 \mathrm{e}-02$ & $6.1085 \mathrm{e}-03$ & $3.5880 \mathrm{e}-03$ & $2.0481 \mathrm{e}-03$ & $1.1453 \mathrm{e}-03$ \\
$10^{-6}$ & $5.8788 \mathrm{e}-03$ & $3.4715 \mathrm{e}-03$ & $2.0256 \mathrm{e}-03$ & $1.1558 \mathrm{e}-03$ & $6.4569 \mathrm{e}-04$ \\
$10^{-7}$ & $3.7402 \mathrm{e}-03$ & $2.0425 \mathrm{e}-03$ & $1.1544 \mathrm{e}-03$ & $6.5221 \mathrm{e}-04$ & $3.6521 \mathrm{e}-04$ \\
$10^{-8}$ & $2.7490 \mathrm{e}-03$ & $1.3093 \mathrm{e}-03$ & $6.8553 \mathrm{e}-04$ & $3.7354 \mathrm{e}-04$ & $2.0573 \mathrm{e}-04$ \\
$10^{-9}$ & $2.3519 \mathrm{e}-03$ & $9.7032 \mathrm{e}-04$ & $4.4669 \mathrm{e}-04$ & $2.2466 \mathrm{e}-04$ & $1.1870 \mathrm{e}-04$ \\
\hline
\end{tabular}


Another issue of interest which we are investigating is whether the system of equations obtained in [13] by using non-standard finite difference method coincides with the one obtained via the finite element method with numerical integration. This issue is successfully addressed in the case of standard finite difference method $([3,20])$ applied to non-singularly perturbed problems.

\section{References}

[1] R.A. Adams, Sobolev Spaces, Academic Press, New York, 1975.

[2] I. Babuska, Finite element method for domains with corners, Computing 6 (1970), 264273.

[3] P.G. Ciarlet, The Finite Element Method for Elliptic Problems, North-Holland, Amsterdam, 1978.

[4] E.P. Doolan, J.J.H. Miller and W.H.A. Schilders, Uniform Numerical Methods for Problems with Initial and Boundary Layers, Boole Press, Dublin, 1980.

[5] G.J. Fix, S. Gulati and G.I. Wakoff, On the use of singular functions with finite element approximations, J. Comp. Phys. 13 (1973) 209-228.

[6] E.C. Gartland, An analysis of a uniformly convergent finite difference/finite element scheme for a model singular-perturbation problem, Math. Comp. 51 (1988) 93-106.

[7] C.Y. Jung and R. Temam, Numerical approximation of two-dimensional convectiondiffusion equations with multiple boundary layers, International Journal of Numerical Analysis and Modeling 2 (2005) 367-408.

[8] M.K. Kadalbajoo and K.C. Patidar, A survey of numerical techniques for solving singularly perturbed ordinary differential equations, Applied Mathematics and Computations 130 (2002) 457-510.

[9] X. Li, C.K. Chan and S. Wang, The finite element method with weighted basis functions for singularly perturbed convection diffusion problems, J. Comp. Phys. 195(2) (2004) 773-789.

[10] V.D. Liseikin, Layer Resolving Grids and Transformations for Singular Perturbation Problems, VSP BV, Utrecht, 2001.

[11] W. Liu and T. Tang, Error analysis for a Galerkin-spectral method with coordinate transformation for solving singularly perturbed problems, Appl. Numer. Math. 38(3) (2001) 315-345.

[12] J.M.-S. Lubuma and K.C. Patidar, Non-standard finite difference methods for dissipative singular perturbation problems, In: L.J.S. Allen, B. Aulbach, S. Elaydi and R. Sacker (Editors), Difference Equations and Discrete Dynamical Systems, (Proceedings of the 9th International Conference on Difference Equations and Applications, University of Southern California, Los Angeles, California, USA, Aug. 2-7, 2004), World Scientific, Singapore, 2005, pp. 185-198. 
[13] J.M.-S. Lubuma and K.C. Patidar, Uniformly convergent non-standard finite difference methods for self-adjoint singular perturbation problems, J. Comput. Appl. Math. 191 (2006) 229-238.

[14] J.M.-S. Lubuma and K.C. Patidar, Contributions to the theory of non-standard finite difference methods and applications to singular perturbation problems. In: R.E. Mickens (editor), Advances in the Applications of Nonstandard Finite Difference Schemes, World Scientific, Singapore, 2005, pp. 513-560.

[15] J.M.-S. Lubuma and K.C. Patidar, Non-standard finite difference methods for singularly perturbed problems possessing oscillatory/layer solutions, Appl. Math. Comput. 187 (2007) 1147-1160.

[16] J.H. Miller (ed), Applications of Advanced Computational Methods for Boundary and Interior Layers, Boole Press, Dublin, 1993.

[17] J.J.H. Miller, E. O'Riordan and G.I. Shishkin, Fitted Numerical Methods for Singular Perturbation Problems, World Scientific, Singapore, 1996.

[18] K.C. Patidar, High order fitted operator numerical method for self-adjoint singular perturbation problems, Appl. Math. Comput. 171(1) (2005) 547-566.

[19] K.C. Patidar, Higher order fitted mesh numerical methods for self-adjoint singular perturbation problems, Internat. J. Comput. Math., to appear.

[20] P.A. Raviart and J.M. Thomas, Introduction àl' Analyse Numérique des Équations Aux Dérivées Partielles, Masson, Paris, 1983.

[21] A.H. Schatz and L.B. Wahlbin, On the finite element method for singularly perturbed reaction diffusion problems in two and one dimension, Math. Comp. 40 (1983) 47-89.

[22] G. Strang and G.J. Fix, An Analysis of the Finite Element Method, Prentice-Hall, Englewood Cliffs, N.J., 1973.

[23] C. Xenophontos, The $h p$-Finite element method for singularly perturbed problems in nonsmooth domains, Numer. Methods Partial Differential Eq. 15 (1999) 63-89. 